\title{
miRNA-708 functions as a tumour suppressor in hepatocellular carcinoma by targeting SMAD3
}

\author{
QI LI ${ }^{1}$, SHENG LI $^{2}$, YAOLU WU ${ }^{3}$ and FENG GAO ${ }^{4}$ \\ ${ }^{1}$ Department of Hepatobiliary Surgery, Yan'an City People's Hospital, Yanan, Shaanxi 716000; \\ ${ }^{2}$ The Second Department of General Surgery, Yulin Second Hospital, Yulin, Shaanxi 719000; Departments of ${ }^{3}$ General Surgery \\ and ${ }^{4}$ Hepatobiliary Surgery, The Affiliated Hospital of Yan'an University, Yanan, Shaanxi 716000, P.R. China
}

Received July 19, 2015; Accepted November 3, 2016

DOI: $10.3892 / \mathrm{ol} .2017 .6429$

\begin{abstract}
Hepatocellular carcinoma (HCC) is the most frequent subtype of primary liver cancer and the third most common cause of cancer-associated mortality worldwide. Previous studies have reported that microRNAs (miRNAs) serve key roles in the carcinogenesis and progression of HCC by regulating gene expression. The present study investigated the expression patterns, biological roles and underlying mechanisms of miRNA-708 (miR-708) in HCC. The expression levels of miR-708 in HCC tissue samples and cell lines were examined. Cell proliferation, migration and invasion assays were used to evaluate the effect of miR-708 on HCC cells. In addition, bioinformatic and western blotting analyses, and dual luciferase reporter assays were performed to investigate the direct gene target of miR-708. The results of the present study demonstrated that miR-708 expression was significantly decreased in HCC tissue samples and cell lines. In addition, the expression level of miR-708 was associated with increased HCC tumour stage. Furthermore, ectopic expression of miR-708 suppressed HCC cell proliferation, migration and invasion. The results of the present study also indicated that miR-708 targets SMAD family member 3 directly in vitro. The results of the present study indicated that miR-708 may be a novel target for future HCC therapy.
\end{abstract}

\section{Introduction}

Hepatocellular carcinoma (HCC), the most frequent subtype of primary liver cancer, accounting for between 85 and $90 \%$ of liver cancer cases, is currently the third leading cause of cancer-associated mortality worldwide (1). In the USA, 35,660 new HCC cases and 24,550 resulting mortalities were recorded in 2015 (2). HCC is prevalent in China and the country accounts

Correspondence to: Professor Qi Li, Department of Hepatobiliary Surgery, Yan'an City People's Hospital, 57 Seven Lane, Yanan, Shaanxi 716000, P.R. China

E-mail: liqi_6199@163.com

Key words: microRNA 708, SMAD family member 3, hepatocellular carcinoma, therapy for $>50 \%$ of all reported cases (3). A number of risk factors have been implicated in HCC carcinogenesis and progression, including alcoholic liver disease, non-alcoholic fatty liver disease, liver cirrhosis, and hepatitis B and C infection (4-6). Despite advances in HCC treatments, including hepatectomy and liver transplantation, the rapid development of HCC results in poor patient prognosis and a five-year survival rate of $<5 \%(7,8)$. The primary challenges in the diagnosis and treatment of HCC include, poor early stage detection, distal metastasis and intrahepatic recurrence following surgery (9). An improved understanding of the underlying molecular mechanisms of HCC initiation and progression may provide novel effective therapeutic targets for the treatment of HCC, and improve prognosis.

MicroRNAs (miRNAs) represent a group of highly conserved, short non-coding RNA molecules of $~ 22$ nucleotides in length (10). miRNAs negatively modulate protein expression through imperfect complementary sequence pairing to the 3' untranslated regions (3'-UTRs) of their target mRNAs, leading to subsequent mRNA degradation or translational repression $(11,12)$. A total of $>1000$ miRNAs have been identified in mammals; however, the biological role of miRNA in carcinogenesis and cancer progression remains unclear $(13,14)$. Increasing evidence suggests that abnormal miRNA expression contributes to alterations in numerous cancer-associated processes, including proliferation, cell cycle progression, apoptosis, survival, migration, invasion and metastasis $(15,16)$. The dysregulation of miRNAs has been reported in various types of human cancer, including human HCC, using miRNA detection systems (17-19). These miRNAs may function as tumour suppressors or oncogenes, and thus may be efficient prognostic biomarkers of HCC tumorigenesis and progression $(20,21)$. miRNAs may also become effective targets in HCC therapy.

The expression and function of miRNA-708 (miR-708) has been studied in several types of human cancer $(22,23)$. The present study investigated the expression patterns, biological roles and underlying mechanisms of miR-708 in HCC. miR-708 was observed to be significantly downregulated in HCC tissue samples and cell lines. Reduced miR-708 expression was significantly associated with increased HCC tumour stage. Furthermore, ectopic miR-708 expression led to decreased cell proliferation, migration and invasion through the direct targeting of SMAD family member 3 (SMAD3). 


\section{Materials and methods}

HCC tissues and ethics statement. HCC and adjacent wild-type tissue sample pairs were obtained from 108 patients with HCC who underwent a hepatectomy at Yan'an City People's Hospital (Yanan, China). None of these patients were treated with neoadjuvant radiotherapy or adjuvant chemotherapy prior to surgery. All tissue specimens were immediately frozen in liquid nitrogen following surgery and stored at $-80^{\circ} \mathrm{C}$. The present study was approved by the Ethics Committee of Yan'an City People's Hospital and informed consent was obtained from all subjects.

Cell lines and cell culture. The human HCC cell lines, HepG2 and SMMC-7721, and the wild-type hepatic cell line L02, were purchased from the Cell Type Culture Collection of the Chinese Academy of Sciences (Shanghai, China). Cells were cultured in Dulbecco's Modified Eagle's medium (DMEM; Gibco; Thermo Fisher Scientific, Inc., Waltham, MA, USA) containing $10 \%$ heat-inactivated foetal bovine serum (FBS; Gibco; Thermo Fisher Scientific, Inc.) at $37^{\circ} \mathrm{C}$ in a humidified atmosphere containing $5 \% \mathrm{CO}_{2}$.

Cell transfection. A mature miR-708 mimic and scrambled miRNA mimic negative control (miR-NC) were purchased from Shanghai GenePharma Co., Ltd. (Shanghai, China). The sequence of the miR-708 mimic was 5'-AAG GAG CUU ACA AUC UAG CUG GG-3'. The sequence of the miR-NC mimic was 5'-UUC UCC GAA CGU GUC ACG UTT-3'. Cells were seeded into six-well plates at between 60 and $70 \%$ confluence and transfected with the miRNA mimics using Lipofectamine ${ }^{\circledR} 2000$ (Invitrogen; Thermo Fisher Scientific, Inc.) reagent according to the manufacturer's protocol.

Reverse transcription-quantitative polymerase chain reaction $(R T-q P C R)$ analysis. Total RNA was extracted from homogenised tissue samples and cell lines using TRIzol ${ }^{\circledR}$ reagent (Invitrogen; Thermo Fisher Scientific, Inc.). cDNA was synthesised using a TaqMan ${ }^{\circledR}$ MicroRNA Reverse Transcription kit (Applied Biosystems; Thermo Fisher Scientific, Inc.) according to the manufacturer's protocol. qPCR was performed using the TaqMan MicroRNA Assay kit (Applied Biosystems; Thermo Fisher Scientific, Inc.) and an Applied Biosystems ${ }^{\circledR}$ 7900HT Fast Real-Time PCR system (Thermo Fisher Scientific, Inc.). The sequences of the primers used were as follows: miR-708 forward, 5'-CGG CGG AAG GAG CTT ACA ATC TA-3' and reverse, 5'-GTG CAG GGT CCG AGG-3'; and U6 forward, 5'-CTC GCT TCG GCA GCA CAT ATA CT-3' and reverse, 5'-ACG CTT CAC GAA TTT GCG TGT C-3'. The thermocycling conditions were as follows: $95^{\circ} \mathrm{C}$ for $10 \mathrm{~min}$; 40 cycles of denaturation at $95^{\circ} \mathrm{C}$ for $15 \mathrm{sec}$ and annealing at $60^{\circ} \mathrm{C}$ for $1 \mathrm{~min}$; followed by a final elongation step at $72^{\circ} \mathrm{C}$ for $10 \mathrm{~min}$. U6 spliceosomal RNA was used as the endogenous control. Fold changes in relative expression were calculated using the $2^{-\Delta \Delta \mathrm{Cq}}$ method (24).

Cell proliferation assay. The Cell Counting Kit-8 (CCK-8; Dojindo Molecular Technologies, Inc., Kumamoto, Japan) assay was used to verify the effect of miR-708 expression in HCC on cell proliferation. A total of 3000 transfected cells
(100 $\mu \mathrm{l})$ were seeded into each well of a 96-well plate. The cells were subsequently incubated at $37^{\circ} \mathrm{C}$ for $24,48,72$ and $96 \mathrm{~h}$ and at each time point the CCK-8 assay was performed. A total of $10 \mu \mathrm{l}$ CCK- 8 reagent was added and incubated at $37^{\circ} \mathrm{C}$ for an additional $2 \mathrm{~h}$. The absorbance was determined at a wavelength of $450 \mathrm{~nm}$ using a microplate reader (Thermo Fisher Scientific, Inc.).

Cell migration and invasion assays. Transwell ${ }^{\circledR}$ chambers (8 $\mu \mathrm{m}$; Corning Incorporated, Corning, NY, USA) and Matrige ${ }^{\circledR}$ (BD Biosciences, Franklin Lakes, NJ, USA)-coated Transwell chambers were inserted into 24-well plates and used in cell migration and invasion assays, respectively. A total of $1 \times 10^{5}$ transfected cells $(300 \mu \mathrm{l})$ in FBS-free DMEM were added to the upper Transwell chamber. A total of $500 \mu \mathrm{l}$ DMEM containing 20\% FBS was added to the lower Transwell chamber. Cells were incubated at $37^{\circ} \mathrm{C}$ for $12 \mathrm{~h}$ (migration assay) and $24 \mathrm{~h}$ (invasion assay). Subsequently, remaining cells in the upper chamber were removed using a cotton-tipped swab. Migratory and invasive cells in the lower chamber were fixed with $100 \%$ methanol, stained with $0.5 \%$ crystal violet (Sigma-Aldrich; Merck Millipore, Darmstadt, Germany) and washed with PBS. Migratory and invasive cells in five randomly selected visual fields were counted using a light microscope.

Western blotting. Total protein was isolated from transfected cells $72 \mathrm{~h}$ following transfection using radioimmunoprecipitation assay buffer (Beyotime Institute of Biotechnology, Haimen, China) containing protease and phosphatase inhibitors. The protein concentration was determined using a Bicinchoninic Acid Protein Assay kit (Beyotime Institute of Biotechnology). Equal masses of total protein $(20 \mu \mathrm{g})$ were separated using SDS-PAGE on a $10 \%$ gel (Beyotime Institute of Biotechnology) and transferred to polyvinylidene difluoride membranes (EMD Millipore, Billerica, MA, USA). Following blocking in $10 \%$ skimmed milk at room temperature for $2 \mathrm{~h}$, the membranes were incubated with rabbit anti-human monoclonal SMAD3 antibody (1:1,000; cat. no. 9523s; Cell Signaling Technology, Inc., Danvers, MA, USA) and mouse anti-human monoclonal $\beta$-actin antibody $(1: 1,000$; cat. no. sc-47778; Santa Cruz Biotechnology, Inc., Dallas, TX, USA) at room temperature for $4 \mathrm{~h}$. The membranes were subsequently washed five times with TBS and Tween 20 and incubated with an anti-rabbit (1:2,000; cat. no. sc-2004; Santa Cruz Biotechnology, Inc.) or anti-mouse (1:2,000; cat. no. sc-2005; Santa Cruz Biotechnology, Inc.) horseradish peroxidase-conjugated secondary antibody for $2 \mathrm{~h}$ at room temperature. Protein bands were detected using the Pierce ${ }^{\mathrm{TM}}$ ECL Western Blotting Substrate (Pierce Biotechnology, Inc., Rockford, IL, USA), and Quantity One ${ }^{\circledR}$ software (version 4.62; Bio-Rad Laboratories, Inc., Hercules, CA, USA).

Dual luciferase reporter assay. Dual luciferase reporter assays were used to confirm whether SMAD3 is a direct target of miR-708. HCC cells were seeded into 24-well plates at a density of between 50 and $60 \%$ confluence, and transfected with the miRNA mimics and plasmids containing the wild-type SMAD3 3'-UTR (pMIR-SMAD3-3'UTR Wt) or a mutated SMAD3 3'-UTR (pMIR-SMAD3-3'UTR Mut; both GenePharma Co., Ltd.) using Lipofectamine 2000. A total of 

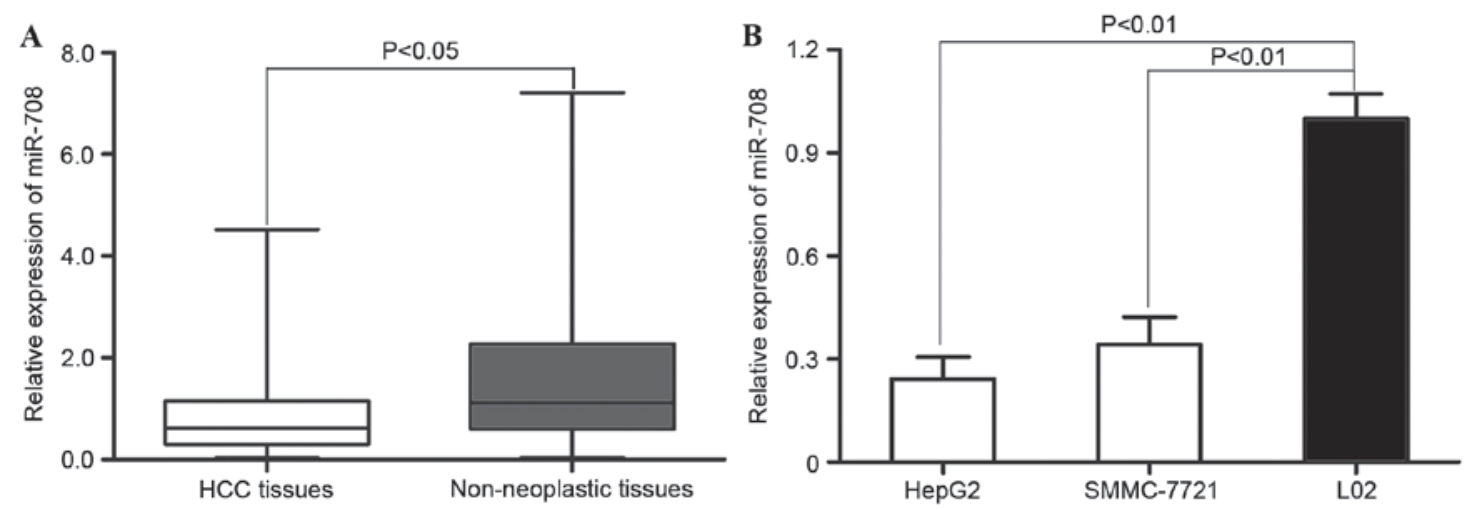

Figure 1. Evaluation of miR-708 expression in HCC tissue samples and cell lines. (A) miR-708 expression in HCC tissue samples was significantly decreased compared with the non-neoplastic tissue samples. (B) miR-708 expression was significantly decreased in the HepG2 and SMMC-7721 cell lines compared with the wild-type L02 cell line. Data normalized to U6 spliceosomal RNA. Data are presented as the mean \pm standard deviation. miR, microRNA; HCC, hepatocellular carcinoma.

$48 \mathrm{~h}$ following transfection, a Dual-Luciferase Reporter Assay system (cat. no. E1910; Promega Corporation, Madison, WI, USA) was used to determine firefly and Renilla luciferase activities. Renilla luciferase activity was used as the internal control.

Statistical and bioinformatic analysis. The putative miR-708 target genes were predicted by bioinformatic analysis using the miRanda database (www.microrna.org). Values are presented as the mean \pm standard deviation of triplicate data and were compared using the Student's $t$-test. Statistical analysis was performed using SPSS (version 17; SPSS, Inc., Chicago, IL, USA). $\mathrm{P}<0.05$ was considered to indicate a statistically significant difference.

\section{Results}

miR-708 is downregulated in HCC tissues and cell lines. To evaluate the biological role of miR-708 in human HCC, RT-qPCR analysis was performed to measure miR-708 expression in HCC and adjacent wild-type tissue samples. As shown in Fig. 1A, miR-708 expression was significantly decreased in HCC tissue samples compared with the adjacent wild-type tissue samples $(\mathrm{P}=0.023)$. In addition, miR-708 expression in the HCC cell lines HepG2 and SMMC-7721, and normal hepatic cell line L02, was analysed. As shown in Fig. 1B, miR-708 was also significantly decreased in the HCC cell lines compared with the wild-type cell line (HepG2, $\mathrm{P}=0.012$; SMMC-7721, $\mathrm{P}=0.017)$. These results suggest that miR-708 is involved in the regulation of HCC malignancy.

Association between miR-708 expression and HCC tumour stage. The association between decreased miR-708 expression and HCC tumour stage was investigated. Statistical analysis demonstrated that miR-708 expression in early (I-II) and advanced (III-IV) tumour stages was significantly decreased compared with the non-neoplastic tissues ( $\mathrm{P}=0.034$; Fig. 2A; $\mathrm{P}=0.026$; Fig. 2B). In addition, significantly decreased miR-708 expression was observed in advanced stage HCC tissue samples compared with the early stage HCC tissue samples $(\mathrm{P}=0.030$; Fig. 2C). These results indicate that decreased
miR-708 expression level is associated with increased HCC tumour stage.

Increased miR-708 expression inhibits HCC cell proliferation. To investigate the role of miR-708 in HCC, a miR-708 mimic was transfected into HepG2 and SMMC-7721 cells. Following transfection, RT-qPCR was performed to evaluate the transfection efficiency, and miR-708 expression was observed to be significantly increased in miR-708-transfected HepG2 $(\mathrm{P}=0.00000013)$ and SMMC-7721 $(\mathrm{P}=0.00000036)$ cells compared with the miR-NC-transfected cells (Fig. 3A). Cell proliferation assays were performed to assess the effect of miR-708 expression on cell proliferation. As shown in Fig. 3B, miR-708 overexpression inhibited cell proliferation in HepG2 and SMMC-7721 cells compared with the miR-NC-transfected cells. A total of $96 \mathrm{~h}$ following transfection, cell proliferation was inhibited by $33.24 \pm 3.5$ and $27.83 \pm 2.7 \%$ in miR-708-transfected HepG2 $(\mathrm{P}=0.018)$ and SMMC-7721 $(\mathrm{P}=0.021)$ cells, respectively.

miR-708 inhibits cell migration and invasion of HCC. Cell migration and invasion assays were performed to investigate the role of miR-708 in the regulation of metastasis in HCC. As shown in Fig. 4, miR-708 overexpression resulted in the significant suppression of migration in HepG2 $(\mathrm{P}=0.033)$ and SMMC-7721 ( $\mathrm{P}=0.024)$ cells compared with the miR-NC-transfected cells. In addition, miR-708 overexpression led to a significant decrease in the number of invasive HepG2 $(\mathrm{P}=0.020)$ and SMMC-7721 $(\mathrm{P}=0.026)$ cells compared with the miR-NC-transfected cells (Fig. 4). These observations suggest that miR-708 is a negative regulator of HCC metastasis.

miR-708 directly targets the 3'-UTR of SMAD3. To further understand the underlying mechanism of miR-708, bioinformatic analysis was performed and a number of candidate target genes were identified. SMAD3, an oncogene in numerous types of cancer $(25,26)$, was selected for further study due to the putative miR-708 target sequences contained within the SMAD3 3'-UTR (Fig. 5A). The dual luciferase reporter assay was performed to evaluate the interaction between miR-708 and the SMAD3 3'-UTR. As shown in Fig. 5B, miR-708 

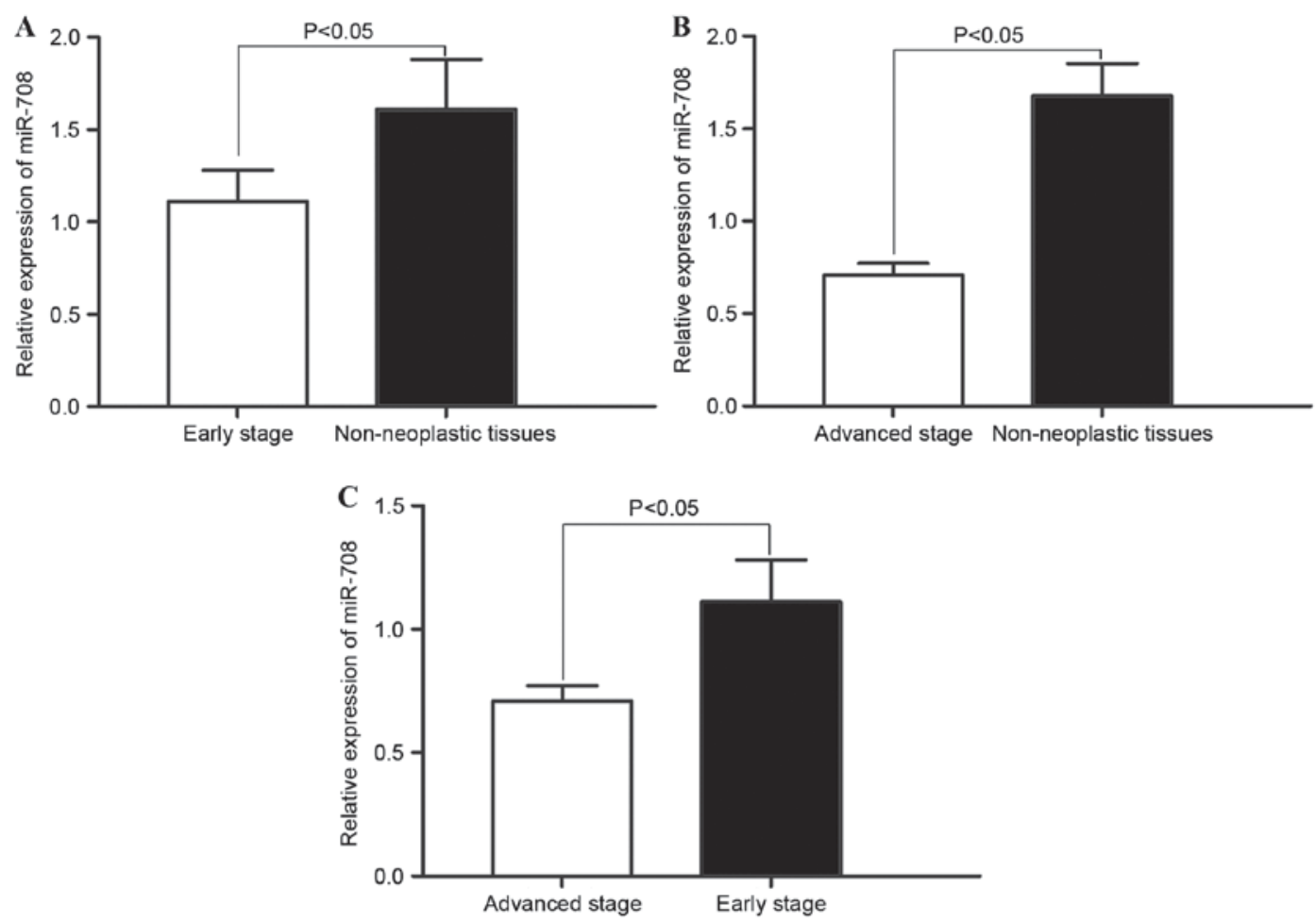

Figure 2. Decreased miR-708 expression is associated with increased HCC tumour stage. miR-708 expression is decreased in (A) early and (B) advanced stage HCC tissue samples compared with non-neoplastic tissue samples. (C) miR-708 expression is decreased in advanced stage HCC tissue samples compared with early stage HCC tissue samples. Data normalized to U6 spliceosomal RNA. Data are presented as the mean \pm standard deviation. miR, microRNA; HCC, hepatocellular carcinoma.
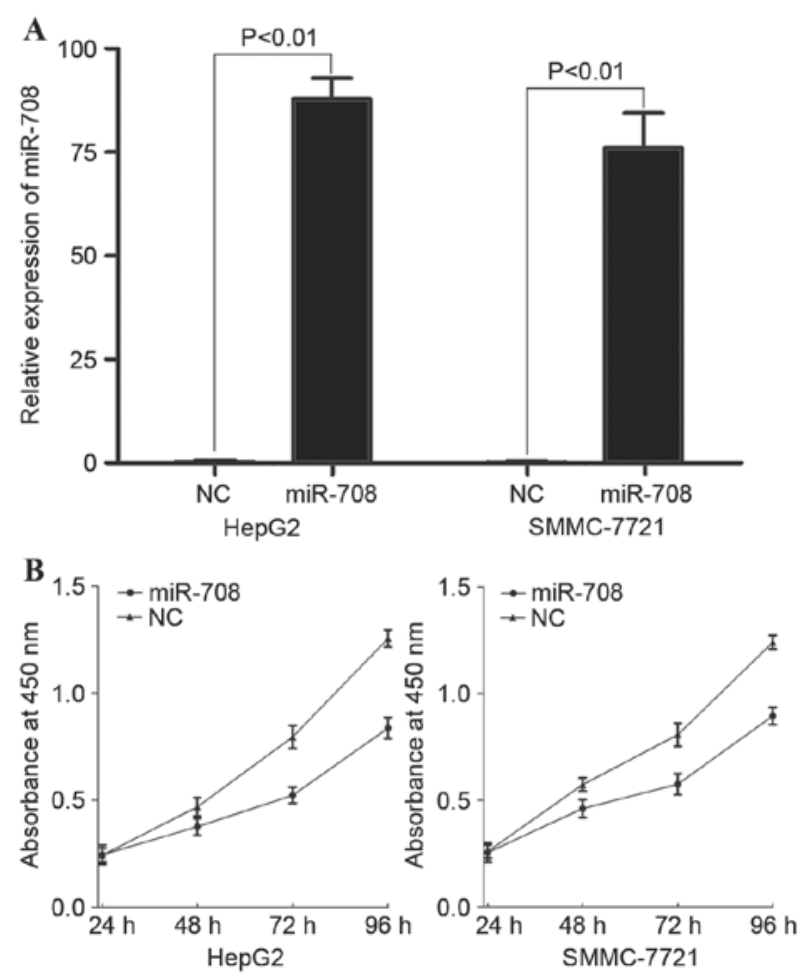

Figure 3. Increased miR-708 expression inhibited cell proliferation in HCC cell lines. (A) miR-708 expression was upregulated in HepG2 and SMMC-7721 cells following transfection with an miR-708 mimic, compared with NC-transfected cells. (B) Increased miR-708 expression decreased cell proliferation in miR-708-transfected HepG2 and SMMC-7721 cells compared with the NC-transfected cells. Data normalized to U6 spliceosomal RNA. Data are presented as the mean \pm standard deviation. miR, microRNA; $\mathrm{HCC}$, hepatocellular carcinoma; NC, negative control microRNA. overexpression significantly decreased luciferase activity in the wild-type SMAD3 3'-UTR-transfected HepG2 $(\mathrm{P}=0.019)$ and SMMC-7721 ( $\mathrm{P}=0.022)$ cells compared with the cells transfected with the mutated SMAD3 3'-UTR. These results suggest that the 3'-UTR of SMAD3 is directly targeted by miR-708.

miR-708 suppresses SMAD3 protein expression in HCC cells. To evaluate the effect of miR-708 expression on SMAD3 protein expression, western blotting was performed in HepG2 and SMMC-7721 cells following miRNA transfection. The results demonstrated that SMAD3 was significantly downregulated in the miR-708-overexpressing HepG2 $(\mathrm{P}=0.013)$ and SMMC-7721 $(\mathrm{P}=0.017)$ cells compared with the miR-NC-transfected cells (Fig. 6). These results suggest that miR-708 negatively regulates SMAD3 protein expression by directly binding to the 3'-UTR of SMAD3 mRNA.

\section{Discussion}

Previous studies have indicated that $1 / 2$ of miRNAs are located in the fragile or oncogene-associated regions of chromosomes, suggesting that abnormally expressed miRNAs are associated with carcinogenesis and cancer progression (27). miRNAs regulate gene expression at the post-transcriptional level via sequence-specific binding to the 3'-UTR of target mRNAs (28). A single miRNA is able to regulate various mRNAs $(29,30)$. miRNAs may therefore present a novel therapeutic target in the treatment of human cancer, including HCC $(31,32)$. To investigate the function of miRNAs in carcinogenesis and 

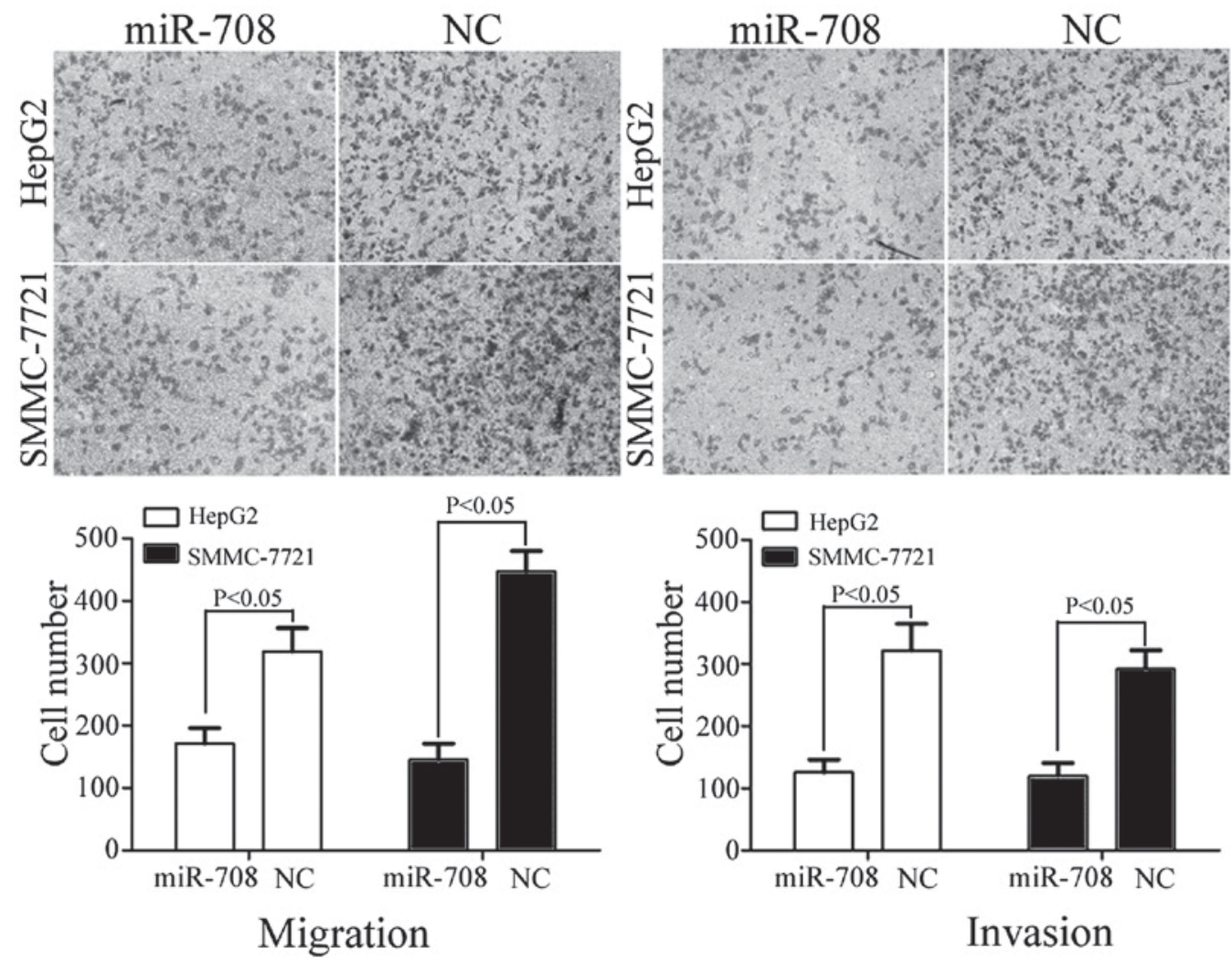

Figure 4. Migration and invasion assays were performed using Transwell ${ }^{\circledast}$ chambers. Ectopic expression of miR-708 suppressed cell migration and invasion in the HepG2 and SMMC-7721 cell lines. Data are presented as the mean \pm standard deviation. miR, microRNA; NC, negative control microRNA.
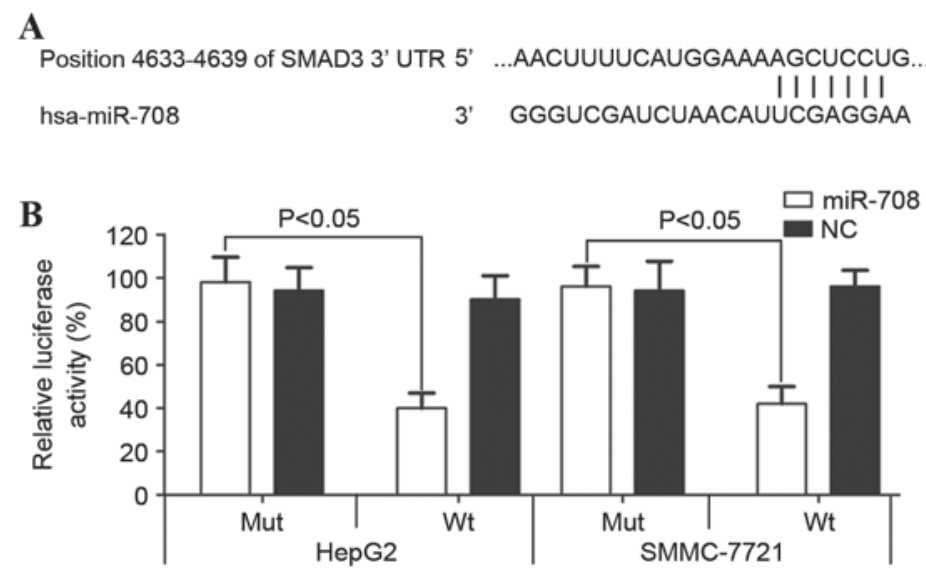

Figure 5. SMAD3 is a direct target gene of miR-708 in vitro. (A) Bioinformatic analysis predicated an interaction between miR-708 and the SMAD3 3'-UTR, between bases 4633 and 4639. (B) Dual luciferase reporter assays revealed that miR-708 inhibited the luciferase activity of the Wt SMAD3 3'-UTR compared with the Mut SMAD3 3'-UTR in HepG2 and SMMC-7721 cells. Data are presented as the mean \pm standard deviation. SMAD3, SMAD family member 3; miR, microRNA; 3'-UTR, 3' untranslated region; Wt, wild-type; Mut, mutated; hsa, Homo sapiens; NC, negative control microRNA.

cancer progression, aberrant miRNA expression must be validated, and the biological role of miRNA in cancer initiation and progression investigated.

miR-708 is dysregulated in multiple types of cancer, and previous studies $(22,23)$ reported that miR-708 was downregulated in renal cell carcinoma and prostate cancer. Guo et al (33) observed that miR-708 expression is low in human glioblastoma. By contrast, miR-708 was observed to be upregulated in non-small cell lung cancer, childhood common precursor B-cell acute lymphoblastic leukaemia and bladder carcinoma $(23,34,35)$. In the present study, it was demonstrated that miR-708 expression was decreased in HCC tissue samples and cell lines. Decreased miR-708 expression was associated with HCC tumour stage. These results suggest that miR-708 expression is tissue specific.

miR-708 also has important roles in several types of human cancer. In human glioblastoma, ectopic expression of miR-708 suppresses cell growth and invasion, and increases apoptosis by negatively regulating multiple target mRNAs, including AKT serine/threonine kinase 1, cyclin D1, matrix metalloproteinase 

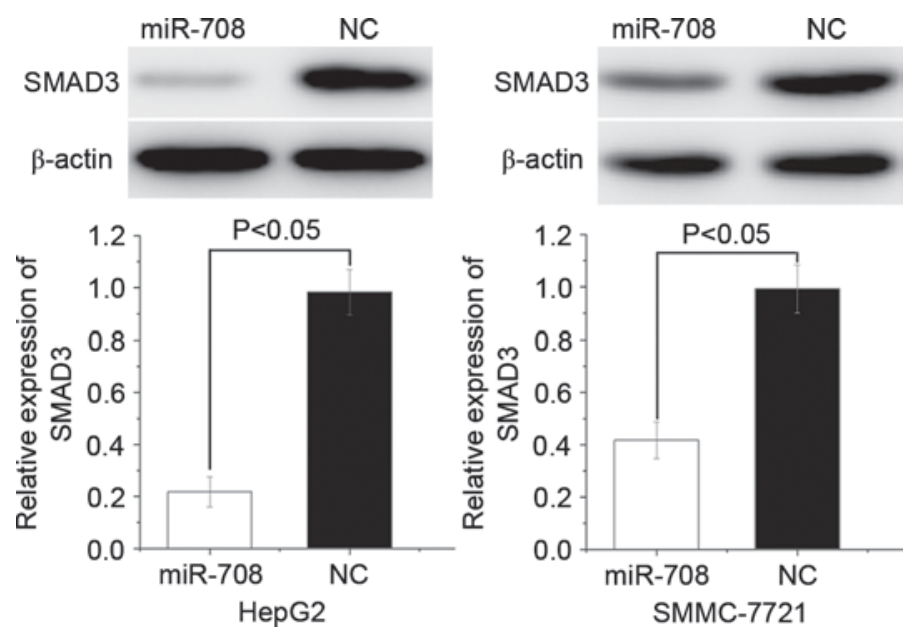

Figure 6. Western blotting was performed to evaluate the effect of miR-708 expression on SMAD3 protein expression in hepatocellular carcinoma cell lines. SMAD3 protein expression was downregulated in HepG2 and SMMC-77213 cells following transfection with miR-708 compared with the NC-transfected cells. Data normalized to $\beta$-actin. Data are presented as the mean \pm standard deviation. miR, microRNA; SMAD3, SMAD family member 3; NC, negative control microRNA.

2, enhancer of zeste 2 polycomb repressive complex 2 subunit, poly (ADP-ribose) polymerase 1 and B-cell lymphoma 2 apoptosis regulator (33). In renal cell carcinoma, ectopic expression of miR-708 represses cell proliferation, clonality and motility, and increases apoptosis by inhibiting zinc finger E-box binding homeobox 2 and BMI1 proto-oncogene polycomb ring finger (22). In prostate cancer, downregulation of miR-708 leads to a significant increase in tumour occurrence and development, through the direct targeting of cluster of differentiation 44 and AKT2 (36). In addition, Li et al (37) reported that miR-708 was downregulated in HCC and was associated with increased Edmondson-Steiner grading and tumour node metastasis stage. In the present study, ectopic expression of miR-708 was demonstrated to repress HCC motility. These findings indicate that miR-708 is a tumour suppressor in human cancer. However, miR-708 has also been demonstrated to function as an oncogene in human cancers. For example, in non-small lung cancer, miR-708 expression is significantly associated with an increased risk of death following adjustment for clinically-relevant factors, including age, gender and tumour stage (23). Furthermore, decreased miR-708 expression has been demonstrated to decrease cell growth and metastasis in vitro by targeting transmembrane 88 mRNA (23). In the present study, ectopic expression of miR-708 suppressed cell proliferation, migration and invasion by inhibiting SMAD3 protein expression. miR-708 serves important roles in multiple types of cancer, and may therefore be a therapeutic target in their treatment.

The identification of cancer-specific miRNAs and their target genes is essential in understanding the role of these miRNAs in HCC carcinogenesis and progression, and in developing novel targeted therapies. In the present study, SMAD3 mRNA was identified to be a direct target of miR-708 in HCC. Bioinformatic analysis predicted SMAD3 to be a target of miR-708 and this was confirmed using luciferase reporter assays, which demonstrated direct binding of miR-708 to the SMAD3 3'-UTR. Western blotting demonstrated that miR-708 reduced SMAD3 protein expression in HCC cell lines. These findings suggest that
miR-708 acts as a tumour suppressor in HCC by directly targeting SMAD3.

The transforming growth factor (TGF)- $\beta$ signalling pathway has an important function in numerous cellular processes, including differentiation, growth, evasion of immunosurveillance, metastasis and neoplasia $(38,39)$. The TGF- $\beta$ signalling pathway is mediated by a type I receptor, a type II receptor and SMAD proteins (40). SMAD3 is the central mediator of the TGF- $\beta$ signalling pathway (40) and functions as an oncogene in numerous types of cancer. In lung carcinoma, decreased SMAD3 expression significantly decreases cell migration and invasion (25). SMAD3 also serves an important role in epithelial-to-mesenchymal transition, which is essential in metastasis (26). In HCC, SMAD3 is upregulated and significantly associated with poor prognosis (41). Therefore, investigation into novel SMAD3-targeted HCC therapies is warranted.

SMAD3 is regulated by multiple miRNAs in numerous types of cancer. In colorectal cancer, miR-140 decreases cell migration and invasion through the regulation of SMAD3 (42). Liu et al (43) reported that miR-34b functions as a tumour suppressor in pancreatic cancer by repressing SMAD3 protein expression. In lung adenocarcinoma, ectopic expression of miR-136 inhibits metastasis by targeting SMAD3 (44). Furthermore, in nasopharyngeal cancer, miR-145 suppresses metastasis through the inhibition of SMAD3 protein expression (45). In the present study, miR-708 negatively regulated SMAD3 protein expression, leading to a subsequent reduction in HCC cell proliferation, migration and invasion. miR-708 may therefore be a novel target of HCC treatment.

In conclusion, the present study demonstrates that miR-708 is significantly downregulated in HCC and associated with increased tumour stage. miR-708 decreases cell proliferation, migration and invasion by directly targeting SMAD3 mRNA in HCC. Identification of miR-708 targets may provide an in-depth understanding of the potential underlying mechanisms of carcinogenesis and progression in HCC. miR-708 may be a novel target for future HCC therapy. 


\section{References}

1. Liu H, Li W, Chen C, Pei Y and Long X: MiR-335 acts as a potential tumor suppressor miRNA via downregulating ROCK1 expression in hepatocellular carcinoma. Tumour Biol 36: 6313-6319, 2015

2. Siegel RL, Miller KD and Jemal A: Cancer statistics, 2015. CA Cancer J Clin 65: 5-29, 2015.

3. Huitzil-Melendez FD, Capanu M, O'Reilly EM, Duffy A, Gansukh B, Saltz LL and Abou-Alfa GK: Advanced hepatocellular carcinoma: Which staging systems best predict prognosis? J Clin Oncol 28: 2889-2895, 2010.

4. Vertemati M, Moscheni C, Petrella D, Lamperti L, Cossa M, Gambacorta M, Goffredi M and Vizzotto L: Morphometric analysis of hepatocellular nodular lesions in $\mathrm{HCV}$ cirrhosis Pathol Res Pract 208: 240-244, 2012.

5. Liang T, Chen EQ and Tang H: Hepatitis B virus gene mutations and hepatocarcinogenesis. Asian Pac J Cancer Prev 14: 4509-4513, 2013.

6. Gao J, Xie L, Yang WS, Zhang W, Gao S, Wang J and Xiang YB: Risk factors of hepatocellular carcinoma-current status and perspectives. Asian Pac J Cancer Prev 13: 743-752, 2012.

7. Yang LY, Fang F, Ou DP, Wu W, Zeng ZJ and Wu F: Solitary large hepatocellular carcinoma: A specific subtype of hepatocellular carcinoma with good outcome after hepatic resection. Ann Surg 249: 118-123, 2009.

8. El-Serag HB and Rudolph KL: Hepatocellular carcinoma: Epidemiology and molecular carcinogenesis. Gastroenterology 132: 2557-2576, 2007.

9. Furuta M, Kozaki KI, Tanaka S, Arii S, Imoto I and Inazawa J: miR-124 and miR-203 are epigenetically silenced tumor-suppressive microRNAs in hepatocellular carcinoma. Carcinogenesis 31: 766-776, 2010.

10. Bartel DP: MicroRNAs: Genomics, biogenesis, mechanism, and function. Cell 116: 281-297, 2004.

11. Iwakawa HO and Tomari Y: Molecular insights into microRNA-mediated translational repression in plants. Mol Cell 52: 591-601, 2013.

12. Engels BM and Hutvagner G: Principles and effects of microRNA-mediated post-transcriptional gene regulation. Oncogene 25: 6163-6169, 2006.

13. Niyazi M, Zehentmayr F, Niemöller OM, Eigenbrod S, Kretzschmar H, Schulze-Osthoff K, Tonn JC, Atkinson M, Mörtl S and Belka C: MiRNA expression patterns predict survival in glioblastoma. Radiat Oncol 6: 153, 2011.

14. Chen G, Lu L, Liu C, Shan L and Yuan D: MicroRNA-377 suppresses cell proliferation and invasion by inhibiting TIAM1 expression in hepatocellular carcinoma. PLoS One 10: e0117714, 2015.

15. Tahara H, Kay MA, Yasui W and Tahara E: MicroRNAs in Cancer: The 22nd Hiroshima Cancer Seminar/the 4th Japanese Association for RNA Interference Joint International Symposium, 30 August 2012, Grand Prince Hotel Hiroshima. Jpn J Clin Oncol 43: 579-582, 2013.

16. Yates LA, Norbury CJ and Gilbert RJ: The long and short of microRNA. Cell 153: 516-519, 2013.

17. Zhang W, Liu K, Liu S, Ji B, Wang Y and Liu Y: MicroRNA-133a functions as a tumor suppressor by targeting IGF-1R in hepatocellular carcinoma. Tumour Biol 36: 9779-9788, 2015.

18. Chen X, Bo L, Zhao X and Chen Q: MicroRNA-133a inhibits cell proliferation, colony formation ability, migration and invasion by targeting matrix metallopeptidase 9 in hepatocellular carcinoma. Mol Med Rep 11: 3900-3907, 2015.

19. Li D, Liu X, Lin L, Hou J, Li N, Wang C, Wang P, Zhang Q, Zhang P, Zhou W, et al: MicroRNA-99a inhibits hepatocellular carcinoma growth and correlates with prognosis of patients with hepatocellular carcinoma. J Biol Chem 286: 36677-36685, 2011.

20. Morishita A and Masaki T: miRNA in hepatocellular carcinoma. Hepatol Res 45: 128-141, 2015.

21. Imbeaud S, Ladeiro Y and Zucman-Rossi J: Identification of novel oncogenes and tumor suppressors in hepatocellular carcinoma. Semin Liver Dis 30: 75-86, 2010.

22. Saini S, Yamamura S, Majid S, Shahryari V, Hirata H, Tanaka Y and Dahiya R: MicroRNA-708 induces apoptosis and suppresses tumorigenicity in renal cancer cells. Cancer Res 71: 6208-6219, 2011.
23. Jang JS, Jeon HS, Sun Z, Aubry MC, Tang H, Park CH, Rakhshan F, Schultz DA, Kolbert CP and Lupu R: Increased miR-708 expression in NSCLC and its association with poor survival in lung adenocarcinoma from never smokers. Clin Cancer Res 18: 3658-3667, 2012.

24. Livak KJ and Schmittgen TD: Analysis of relative gene expression data using real-time quantitative PCR and the 2(-Delta Delta C(T)) Method. Methods 25: 402-408, 2001

25. Tian F, DaCosta Byfield S, Parks WT, Yoo S, Felici A, Tang B, Piek E, Wakefield LM and Roberts AB: Reduction in Smad2/3 signaling enhances tumorigenesis but suppresses metastasis of breast cancer cell lines. Cancer Res 63: 8284-8292, 2003.

26. Zavadil J and Böttinger EP: TGF-beta and epithelial-to-mesenchymal transitions. Oncogene 24: 5764-5774, 2005.

27. Calin GA, Sevignani C, Dumitru CD, Hyslop T, Noch E, Yendamuri S, Shimizu M, Rattan S, Bullrich F, Negrini M and Croce CM: Human microRNA genes are frequently located at fragile sites and genomic regions involved in cancers. Proc Natl Acad Sci USA 101: 2999-3004, 2004.

28. Bartel DP: MicroRNAs: Target recognition and regulatory functions. Cell 136: 215-233, 2009

29. Baek D, Villén J, Shin C, Camargo FD, Gygi SP and Bartel DP: The impact of microRNAs on protein output. Nature 455: 64-71, 2008.

30. Selbach M, Schwanhäusser B, Thierfelder N, Fang Z, Khanin R and Rajewsky N: Widespread changes in protein synthesis induced by microRNAs. Nature 455: 58-63, 2008.

31. Wang Y, Lu Z, Li Y, Ji D, Zhang P, Liu Q and Yao Y: miR-143 inhibits proliferation and invasion of hepatocellular carcinoma cells via down-regulation of TLR2 expression. Xi Bao Yu Fen Zi Mian Yi Xue Za Zhi 30: 1076-1079, 2014 (In Chinese).

32. Yang XW, Zhang LJ, Huang XH, Chen LZ, Su Q, Zeng WT, Li W and Wang Q: miR-145 suppresses cell invasion in hepatocellular carcinoma cells: miR-145 targets ADAM17. Hepatol Res 44: 551-559, 2014

33. Guo P, Lan J, Ge J, Nie Q, Mao Q and Qiu Y: miR-708 acts as a tumor suppressor in human glioblastoma cells. Oncol Rep 30: 870-876, 2013

34. Song T, Zhang X, Zhang L, Dong J, Cai W, Gao J and Hong B: miR-708 promotes the development of bladder carcinoma via direct repression of Caspase-2. J Cancer Res Clin Oncol 139: 1189-1198, 2013.

35. Li X, Li D, Zhuang Y, Shi Q, Wei W and Ju X: Overexpression of miR-708 and its targets in the childhood common precursor B-cell ALL. Pediatr Blood Cancer 60: 2060-2067, 2013.

36. Saini S, Majid S, Shahryari V, Arora S, Yamamura S, Chang I, Zaman MS, Deng G, Tanaka Y and Dahiya R: miRNA-708 control of CD44(+) prostate cancer-initiating cells. Cancer Res 72: 3618-3630, 2012.

37. Li G, Yang F, Xu H, Yue Z, Fang X and Liu J: MicroRNA-708 is downregulated in hepatocellular carcinoma and suppresses tumor invasion and migration. Biomed Pharmacother 73: 154-159, 2015.

38. Kaminska B, Wesolowska A and Danilkiewicz M: TGF beta signalling and its role in tumour pathogenesis. Acta Biochim Pol 52: 329-337, 2005 .

39. Massagué J: TGFbeta in Cancer. Cell 134: 215-230, 2008.

40. Yang YA, Zhang GM, Feigenbaum L and Zhang YE: Smad3 reduces susceptibility to hepatocarcinoma by sensitizing hepatocytes to apoptosis through downregulation of Bcl-2. Cancer Cell 9: 445-457, 2006

41. Kim SH, Ahn S and Park CK: Smad3 and its phosphoisoforms are prognostic predictors of hepatocellular carcinoma after curative hepatectomy. Hepatobiliary Pancreat Dis Int 11: 51-59, 2012.

42. Zhao W, Zou J, Wang B, Fan P, Mao J, Li J, Liu H, Xiao J, Ma W, Wang M, et al: microRNA-140 suppresses the migration and invasion of colorectal cancer cells through targeting Smad3. Zhonghua Zhong Liu Za Zhi 36: 739-745, 2014 (In Chinese).

43. Liu C, Cheng H, Shi S, Cui X, Yang J, Chen L, Cen P, Cai X, Lu Y, Wu C, et al: MicroRNA-34b inhibits pancreatic cancer metastasis through repressing Smad3. Curr Mol Med 13: 467-478, 2013.

44. Yang Y, Liu L, Cai J, Wu J, Guan H, Zhu X, Yuan J, Chen S and Li M: Targeting Smad2 and Smad3 by miR-136 suppresses metastasis-associated traits of lung adenocarcinoma cells. Oncol Res 21: 345-352, 2013.

45. Huang H, Sun P, Lei Z, Li M, Wang Y,Zhang HT and Liu J: miR-145 inhibits invasion and metastasis by directly targeting Smad3 in nasopharyngeal cancer. Tumour Biol 36: 4123-4131, 2015. 